6 Calverley PMA, Anderson JA, Celli B, et al. Salmeterol and fluticasone propionate and survival in chronic obstructive pulmonary disease. N Engl J Med 2007; 356: 775-789.

7 Martinez FJ, Boscia J, Feldman G, et al. Fluticasone furoate/vilanterol (100/25; 200/25 $\mu \mathrm{g})$ improves lung function in COPD: a randomised trial. Respir Med 2013; 107: 550-559.

8 van der Valk P, Monninkhof E, van der Palen J, et al. Effect of discontinuation of inhaled corticosteroids in patients with chronic obstructive pulmonary disease: the COPE study. Am J Respir Crit Care Med 2002; 166: 1358-1363.

9 Wouters EFM, Postma DS, Fokkens B, et al. Withdrawal of fluticasone propionate from combined salmeterol/ fluticasone treatment in patients with COPD causes immediate and sustained disease deterioration: a randomised controlled trial. Thorax 2005; 60: 480-487.

\title{
Bacteraemia in outpatients with community-acquired pneumonia
}

\author{
To the Editor:
}

Community-acquired pneumonia (CAP) is a frequent cause of morbidity and mortality worldwide. Approximately $20 \%$ of CAP patients require hospital admission $[1,2]$, leaving a large percentage of patients treated in the community as outpatients. In addition, bacteraemia is associated with worse outcomes, such as a higher prevalence of intensive care unit (ICU) admission and longer length of stay. Current international guidelines [1, 3, 4] do not recommend blood cultures as part of routine microbiological tests in patients managed in the community. However, there is little information regarding the prevalence and clinical impact of bacteraemia in CAP patients treated outside the hospital. Previous studies reported incidences of $2.1-7 \%$, respectively [5-8]. However, few data exist regard predictive factors and the clinical outcomes associated with positive blood cultures in this population [8]. The current study aimed to determine the predictive factors and the clinical outcomes of bacteraemic outpatients with CAP, and describe their clinical, epidemiological and microbiological characteristics.

We performed a prospective study in Hospital Clinic of Barcelona, Barcelona, Spain. The study population consisted of adults aged 16 years or older, consecutively admitted to the emergency room with a diagnosis of CAP and discharged (within $12 \mathrm{~h}$ ) for ambulatory treatments, from January 2000 to July 2014. We analysed all patients with available blood cultures (we drew four samples, two from the arm (anaerobic and aerobic); the following vials were used: the resin-containing BACTEC plus Aerobic/F and BACTEC plus Anaerobic/F, and the non-resin-containing BACTEC Standard/10 Aerobic/F and BACTEC Lytic/10 Anaerobic/F (BD, Madrid, Spain)). Contaminants were considered as a negative blood culture. Exclusion criteria are presented in the footnote of table 1.

During the study period, 6086 patients were admitted with a diagnosis of CAP. Of these, 687 (11\% of total CAP) were treated as outpatients. $315(46 \%)$ patients had no blood cultures. The study population therefore comprised a total of $372(54 \%)$ patients treated as outpatients; 26 (7\%) out of these 372 had bacteraemia. Both groups had similar baseline characteristics (table 1), except for bacteraemic patients having a higher proportion aged 50 years or over and pleuritic pain on presentation of pneumonia, and higher median levels of C-reactive protein $\left(24.9 \mathrm{mg} \cdot \mathrm{dL}^{-1}\right.$ (interquartile range $15.4-29.0 \mathrm{mg} \cdot \mathrm{dL}^{-1}$ ) versus $\left.12.4 \mathrm{mg} \cdot \mathrm{dL}^{-1}\left(6.7-21.1 \mathrm{mg} \cdot \mathrm{dL}^{-1}\right) ; \mathrm{p}=0.001\right)$ and white blood cell count on admission. There were no differences between the two groups on admission according to the CURB-65 (confusion, urea $>7 \mathrm{mmol} \cdot \mathrm{L}^{-1}$, respiratory rate $\geqslant 30$ breaths per $\mathrm{min}$, systolic blood pressure $<90 \mathrm{mmHg}$ or diastolic blood pressure $\leqslant 60 \mathrm{mmHg}$, age $\geqslant 65$ years) and Pneumonia Severity Index (PSI) scores. None of the patients with bacteraemia was readmitted, presented treatment failure or died. 30 -day mortality was $0 \%$.

Aetiological diagnoses were established in 124 (33\%) cases. The most frequently isolated pathogen was Streptococcus pneumoniae (62 (50\%) patients). The remaining microorganisms are presented in the footnote of table 1. Microorganisms isolated in bacteraemic cases were S. pneumoniae (24 (92\%) patients) and Haemophilus influenzae (two (8\%) patients). The most frequent serotypes in bacteraemic cases were 1, $19 \mathrm{~A}, 3,6 \mathrm{~A}, 7 \mathrm{~F}, 11 \mathrm{~A}, 10 \mathrm{~A}$ and 13 .

Patients with bacteraemic CAP received the following antibiotics: fluoroquinolone monotherapy $(\mathrm{n}=15,58 \%)$, a $\beta$-lactam plus a macrolide $(n=6,24 \%)$, a $\beta$-lactam plus a fluoroquinolone $(n=1,4 \%)$, a fluoroquinolone plus 
TABLE 1 Clinical characteristics of bacteraemic and nonbacteraemic community-acquired pneumonia (CAP) outpatients, and significant univariate and multivariate logistic regression analyses of predictors for bacteraemia in outpatients

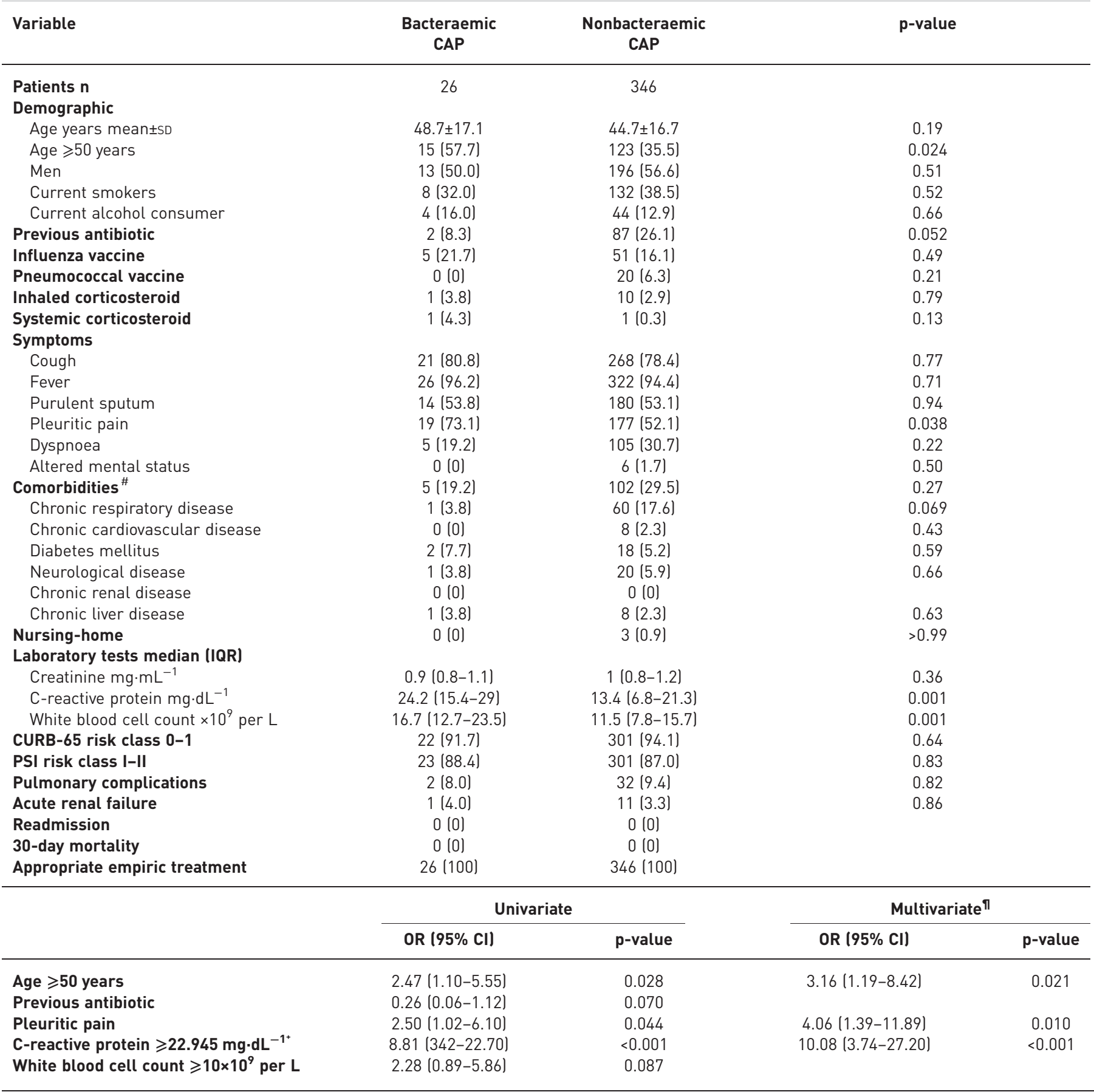

Data are presented as $\mathrm{n}(\%)$ unless otherwise stated. Percentages were calculated using nonmissing data. Exclusion criteria were: 1) unavailable blood culture; 2) severe immunosuppression (AIDS, chemotherapy or immunosuppressive drugs (e.g. oral corticosteroid $\geqslant 10 \mathrm{mg}$ prednisone or equivalent per day for $\geqslant 2$ weeks)); 3) active tuberculosis; and 4) cases with a confirmed alternate diagnosis. Microbial aetiology: Mycoplasma pneumoniae $(n=15,12 \%)$; respiratory viruses $(n=10,8 \%)$; Legionella pneumophila $(n=10,8 \%)$; Coxiella burnetii $(n=5,4 \%)$, Chlamydophila pneumoniae $(n=5,4 \%)$; and Haemophilus influenzae $(n=5,4 \%)$. More than one causative agent was found in nine $(7 \%)$ patients; Streptococcus pneumoniae was the most prevalent microorganism involved in mixed infections (four (44\%) out of nine). Serotypes in 11 bacteraemic cases were: 1 ( $n=3,27 \%$ ); 19A ( $\mathrm{n}=1$, $9 \%) ; 3(n=1,9 \%) ; 6 A(n=1,9 \%) ; 7 F(n=2,18 \%) ; 11 A(n=1,9 \%) ; 10 A(n=1,9 \%)$; and $13(n=1,9 \%)$. IQR: interquartile range; CURB-65: confusion, urea $>7 \mathrm{mmol} \cdot \mathrm{L}^{-1}$, respiratory rate $\geqslant 30$ breaths per $\mathrm{min}$, systolic blood pressure $<90 \mathrm{mmHg}$ or diastolic blood pressure $\leqslant 60 \mathrm{mmHg}$, age $\geqslant 65$ years; PSI: Pneumonia Severity Index. "\#: patients could have more than one comorbidity; ๆ: Hosmer-Lemeshow goodness-of-fit test, $p=0.49 ;{ }^{+}$: optimal cut-off value to predict bacteraemic CAP using receiver operating characteristic curves. 
a macrolide $(n=1,4 \%), \beta$-lactam monotherapy $(n=1,4 \%)$ and other combinations $(n=2,8 \%) .21(81 \%)$ out of the 26 bacteraemic patients $(81 \%)$ received a single dose of intravenous antibiotic in the emergency department before discharge (ceftriaxone $(n=10)$, levofloxacin $(n=10)$ and amoxicillin/clavulanate $(n=1)$ ). Intravenous antibiotics were always administered after blood cultures were taken.

There were no differences in empiric antibiotic therapy between nonbacteraemic and bacteraemic CAP. There were no cases with inappropriate initial empiric antibiotic treatment in patients with bacteraemia.

Age $\geqslant 50$ years, pleuritic pain and C-reactive protein $\geqslant 22.945 \mathrm{mg} \cdot \mathrm{dL}^{-1}$ were risk factors for bacteraemia in the multivariate analysis. In the presence of these three risk factors, the probability of bacteraemia was $49.4 \%$ and without any of these risk factors was $0.7 \%$ (table 2).

Bacteraemia is a complication of hospitalised CAP, with a prevalence that ranges from $7 \%$ to $20 \%$ [9-13]. Little is known about bacteraemic CAP in nonadmitted patients. In our study, the prevalence of these cases was $7 \%$ of the total bacteraemic cases collected over 10 years. In a review of the literature, we found percentages that ranged between $2.1 \%$ and $7 \%[5-8,13]$.

Our study may help in the decision to hospitalise CAP patients (until blood results are available) who can be managed outside the hospital according to PSI and CURB-65, but who may have a positive blood culture.

Conversely, an important finding of our study was that bacteraemic patients treated in the emergency department and cared for outside the hospital did not show treatment failure, re-admission or death. It is important to remark that the majority of these patients $(81 \%)$ received one intravenous dose of antibiotic (ceftriaxone or levofloxacin) before discharge. Falguera et al. [9] found that chronic liver disease, pleuritic pain and vital sign abnormalities were predictors of bacteraemia. A high level of C-reactive protein was also found to be a predictive factor for bacteraemia. LeE et al. [14] found that systolic blood pressure $<90 \mathrm{mmHg}$, heart rate $\geqslant 125$ beats per min, body temperature $<35^{\circ} \mathrm{C}$ or $>40^{\circ} \mathrm{C}$, white blood cell count $<4000$ or $>12000$ per $\mathrm{mm}^{3}$, platelet count $<130$ per $\mathrm{mm}^{3}$, albumin $<3.3 \mathrm{~g} \cdot \mathrm{dL}^{-1}$ and C-reactive protein $\geqslant 17 \mathrm{mg} \cdot \mathrm{dL}^{-1}$ were associated with bacteraemia. METERSKY et al. [11] found that liver disease, recent antibiotic treatment, temperature $<35^{\circ} \mathrm{C}$ or $\geqslant 40^{\circ} \mathrm{C}$, blood urea nitrogen $\geqslant 30 \mathrm{mg} \cdot \mathrm{dL}^{-1}$, sodium $<1.3 \mathrm{mg} \cdot \mathrm{dL}^{-1}$, and white blood cell count $<5000$ or $>20000$ per $\mathrm{mm}^{3}$ were associated with bacteraemia. BENENSON et al. [8], in a retrospective study, found that oxygen saturation $<90 \%$, serum sodium $<130 \mathrm{mg} \cdot \mathrm{dL}^{-1}$ and respiratory rate $>30$ breaths per min were factors associated with a positive blood culture in a cohort of hospitalised patients with CAP. In another retrospective study [7] of 414 cases, positive blood cultures were identified in $7 \%$ of cases but no predictors were provided. In this study, antibiotic therapy was modified only in $15(3.6 \%)$ cases with positive blood culture.

As expected, in most cases, bacteraemia in nonadmitted CAP patients was caused by S. pneumoniae; however, we also observed two cases of $H$. influenzae. The majority of the serotypes found were covered by the 23 -valent pneumococcal polysaccharide vaccine $(82 \%)$ and by the 13 -valent pneumococcal conjugate vaccine $(73 \%)$.

What we learned from our study is that there are certain high-risk patients who are discharged from the emergency department and have a high risk of bacteraemia, and they can be identified using the risk factors we found. Therefore, we recommend using risk factors, in the absence of drawing blood cultures, to identify patients who could benefit from a dose of $i$.v. antibiotics before being sent home. However, an important point is that blood cultures have a main value regarding the observation of microbial resistance development. The major strength of our study is the large number of patients included over a long period of consecutively collecting cases of CAP arriving in the emergency department. A limitation is that blood cultures were not obtained in all nonhospitalised patients. Thus, we do not know the overall prevalence of bacteraemia in this population.

TABLE 2 Probability of bacteraemia in outpatients with community-acquired pneumonia

\section{None}

Age $\geqslant 50$ years 
Due to these limitations and because this is a single-centre study, we recommend validating our findings in other CAP cohorts.

The data in this study extend and support the recommendation of the Infectious Diseases Society of America/American Thoracic Society guidelines [3] for non-ICU patients, who were sent home, in that blood cultures added no useful data. Therefore, our findings extend to those discharged the lack of a need to draw blood samples for cultures. However, there are risk factors (three that we identified) for bacteraemia in those discharged and in this population, we believe, based on the good outcome, that the risk factors can be used not to recommend drawing blood for cultures but rather to assure that these patients will have a more strict follow-up.

0 @ERSpublications

CAP patients who are discharged but have a high risk of bacteraemia can be identified using risk factors http://ow.ly/TcMiq

Catia Cillóniz ${ }^{1}$, Albert Gabarrús ${ }^{1}$, Jordi Almirall ${ }^{2}$, Rosanel Amaro ${ }^{1}$, Mariano Rinaudo ${ }^{1}$, Chiara Travierso ${ }^{3}$, Michael Niederman ${ }^{4}$ and Antoni Torres ${ }^{1}$

${ }^{1}$ Dept of Pneumology, Institut Clinic del Tórax, Hospital Clinic of Barcelona - Institut d'Investigacions Biomèdiques August Pi i Sunyer (IDIBAPS), University of Barcelona (UB) - SGR 911- Ciber de Enfermedades Respiratorias (Ciberes), Barcelona, Spain. ${ }^{2}$ Critical Care Unit, Hospital de Mataró, Universitat Autònoma de Barcelona, Ciberes, Barcelona, Spain. ${ }^{3}$ Dipartimento di Fisiopatologia Medico-Chirurgica e dei Trapianti, Fondazione IRCCS Ca' GrandaOspedale Maggiore Policlinico, Università degli Studi di Milano, Milan, Italy. ${ }^{4}$ Dept of Medicine, Winthrop University Hospital, Mineola, NY, USA.

Correspondence: Antoni Torres, Department of Pneumology, Hospital Clinic of Barcelona, Villarroel 170, Barcelona 08036, Spain. E-mail: atorres@clinic.ub.es

Received: June 192015 | Accepted after revision: Sept 192015 | First published online: Nov 052015

Support statement: This work was supported by CibeRes (grant CB06/06/0028), 2009 Support to Research Groups of Catalonia 911 and IDIBAPS.

Conflict of interest: None declared.

\section{References}

1 Lim WS, Baudouin SV, George RC, et al. BTS guidelines for the management of community acquired pneumonia in adults: update 2009. Thorax 2009; 64: Suppl 3, iii1-iii55.

2 Waterer GW, Wunderink RG. The influence of the severity of community-acquired pneumonia on the usefulness of blood cultures. Respir Med 2001; 95: 78-82.

3 Mandell LA, Wunderink RG, Anzueto A, et al. Infectious Diseases Society of America/American Thoracic Society consensus guidelines on the management of community-acquired pneumonia in adults. Clin Infect Dis 2007; 44: Suppl. 2, S27-S72.

4 Woodhead M, Blasi F, Ewig S, et al. Guidelines for the management of adult lower respiratory tract infections full version. Clin Microbiol Infect 2011; 17: Suppl. 6, E1-E59.

5 Marrie TJ. Blood cultures in ambulatory patients who are discharged from emergency with community-acquired pneumonia. Can J Infect Dis 2004; 15: 21-24.

6 Cilloniz C, Ewig S, Polverino E, et al. Community-acquired pneumonia in outpatients: aetiology and outcomes. Eur Respir J 2012; 40: 931-938.

7 Kennedy M, Bates DW, Wright SB, et al. Do emergency department blood cultures change practice in patients with pneumonia? Ann Emerg Med 2005; 46: 393-400.

8 Benenson RS, Kepner AM, Pyle DN, et al. Selective use of blood cultures in emergency department pneumonia patients. J Emerg Med 2007; 33: 1-8.

9 Falguera M, Trujillano J, Caro S, et al. A prediction rule for estimating the risk of bacteremia in patients with community-acquired pneumonia. Clin Infect Dis 2009; 49: 409-416.

10 Muller F, Christ-Crain M, Bregenzer $\mathrm{T}$, et al. Procalcitonin levels predict bacteremia in patients with community-acquired pneumonia: a prospective cohort trial. Chest 2010; 138: 121-129.

11 Metersky ML, Ma A, Bratzler DW, et al. Predicting bacteremia in patients with community-acquired pneumonia. Am J Respir Crit Care Med 2004; 169: 342-347.

12 Bartlett JG, Dowell SF, Mandell LA, et al. Practice guidelines for the management of community-acquired pneumonia in adults. Infectious Diseases Society of America. Clin Infect Dis 2000; 31: 347-382.

13 Campbell SG, Marrie TJ, Anstey R, et al. Utility of blood cultures in the management of adults with community acquired pneumonia discharged from the emergency department. Emerg Med J 2003; 20: 521-523.

14 Lee J, Hwang SS, Kim K, et al. Bacteremia prediction model using a common clinical test in patients with community-acquired pneumonia. Am J Emerg Med 2014; 32: 700-704. 